\title{
3D cardiac navigation with rapid multi shot EPI
}

\author{
Aaron T Hess ${ }^{1 *}$, André J van der Kouwe ${ }^{2}$, Stefan Neubauer ${ }^{1}$, Matthew D Robson ${ }^{1}$ \\ From 15th Annual SCMR Scientific Sessions \\ Orlando, FL, USA. 2-5 February 2012
}

\section{Summary}

To assess a rapid 3D multishot EPI acquisition as an improved cardiac respiratory navigator.

\section{Background}

3D EPI navigators are a robust real-time brain navigation tool [1], they allow rapid online reconstruction and image registration $(<80 \mathrm{~ms})$. A thoracic EPI volume can be acquired in $200 \mathrm{~ms}$, thus allowing real-time navigation. An analysis of the EPI navigators' stability and variance when registering the heart is presented.

\section{Methods}

EPI parameters were: flip angle $2^{\circ}$, FOV (v1) 332 x $221 \times$ $144 \mathrm{~mm}^{3}$ or (v2 to v4) $400 \times 300 \times 150 \mathrm{~mm}^{3}$, acquisition matrix $48 \times 36 \times 18$, TR $14 \mathrm{~ms}$, TE $6.3 \mathrm{~ms}$, slice partial Fourier 6/8, and bandwidth $3858 \mathrm{~Hz} /$ pixel, acquisition time $200 \mathrm{~ms}$. The registration region of interest (ROI), the heart, was identified using the adjustment volume. The images were reconstructed in real-time and fed into a modified 3D PACE rigid body registration [2] which registered the ROI to that of the first navigator's volume.

Four volunteers (mean age $32+/-7$ years) were scanned on a Siemens 3T. For each, a scan was acquired with 50 navigator volumes, one per R-R interval. Each volunteer held their breath at end expiration for $+/-10$ heart beats, then at end inspiration for $+/-10$ heart beats, repeating this until the end of the scan. A fifth volunteer was instructed to breathe deeply for the entire scan. Finally the navigators' impact on $\mathrm{Mz}$ was measured with a Bloch simulator [3].

\section{Results}

A sample navigator volume and the translations and rotation estimates from one volunteer are shown in the figure. The standard deviation of each motion estimate, calculated as in [4] and by excluding transitions zones, are presented in the table. These measures demonstrate an upper limit on registration variance/stability of 0.6 $\mathrm{mm}$ and $0.5^{\circ}$. The motion estimates for the fifth volunteer, with deep breathing, exceeded $4 \mathrm{~mm}$ or $4^{\circ}$ in all measures. The Bloch simulator shows that the sum effect of the $2^{\circ}$ flip angles reduces the Mz by $0.7 \%$.

\section{Conclusions}

EPI proves to be rapid, reliable and consistent as a heart navigator. Its $2^{\circ}$ flip angle has a minimal effect on the image contrast $(\mathrm{Mz})$. The real-time nature of this navigator would prove particularly beneficial for techniques like spectroscopy, high resolution imaging, and various forms of functional cardiac imaging.

\section{Funding}

This work was supported by the Medical Research Council [G0900883].

\section{Author details MA, USA. \\ Published: 1 February 2012 \\ References \\ 1. Hess : MRM. 2011 \\ 2. Thesen : MRM. 2000. \\ 3. Code : Hargreaves. 2003. \\ 4. Jackson : IJCI. 2009.}

${ }^{1}$ Department of Cardiovascular Medicine, John Radcliffe Hospital, University of Oxford Centre for Clinical Magnetic Resonance Research, Oxford, UK. ${ }^{2}$ Athinoula A. Martinos Center for Biomedical Imaging, MGH, Charlestown,

doi:10.1186/1532-429X-14-S1-W32

Cite this article as: Hess et al:: 3D cardiac navigation with rapid multi shot EPI. Journal of Cardiovascular Magnetic Resonance 2012 14(Suppl 1): W32. 

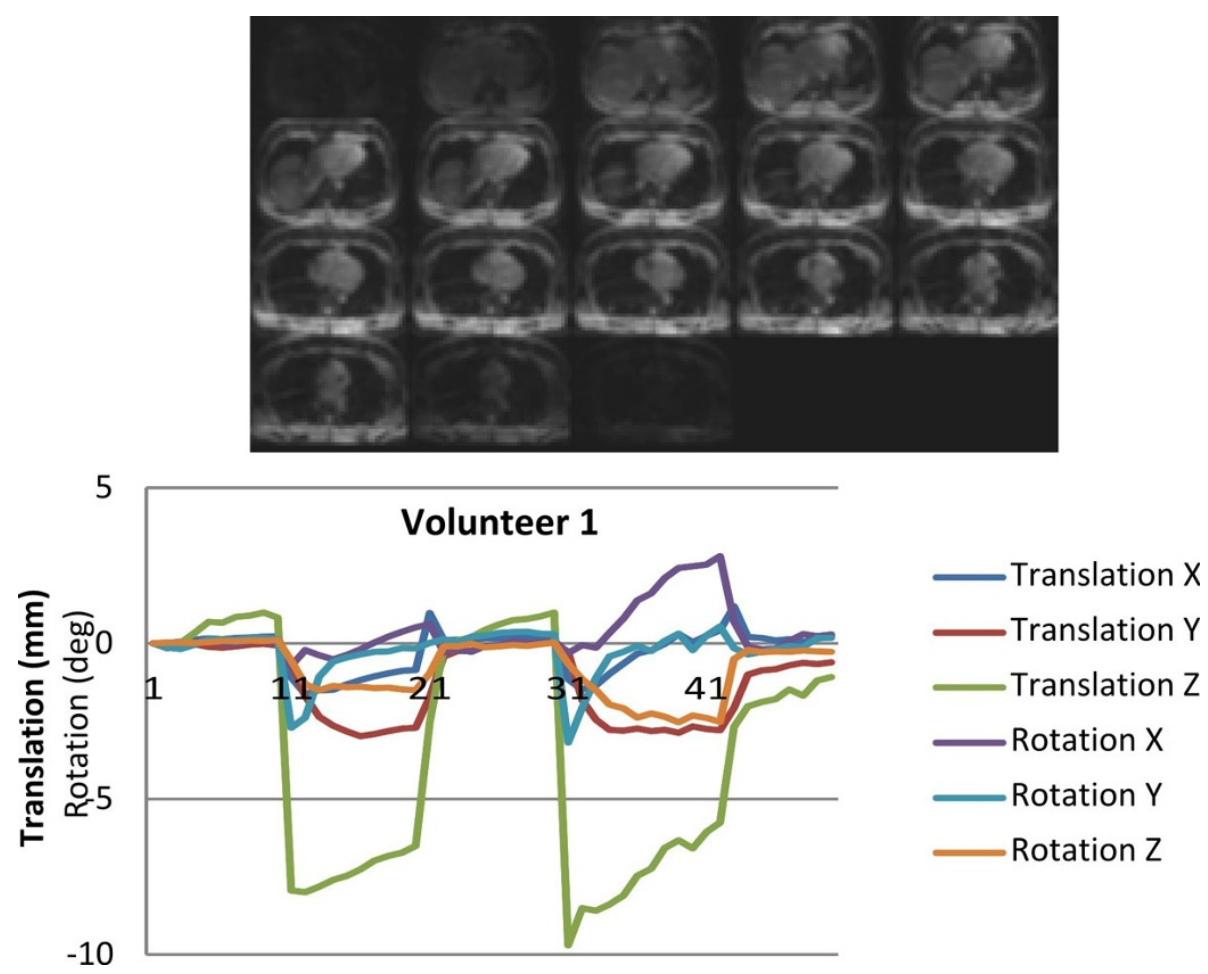

Figure 1 Example navigator volume and registration result from a volunteer

Table 1 Standard deviation of registration during breathhold periods

\begin{tabular}{ccccccc}
\hline Volunteer & \multicolumn{3}{c}{ Translation $(\mathrm{mm})$} & \multicolumn{3}{c}{ Rotation (deg) } \\
\cline { 2 - 7 } & $X$ & $Y$ & $Z$ & $X$ & $Y$ & $Z$ \\
\hline 1 & 0.3 & 0.3 & 0.4 & 0.5 & 0.4 & 0.3 \\
2 & 0.5 & 0.3 & 0.5 & 0.4 & 0.4 & 0.2 \\
3 & 0.3 & 0.3 & 0.6 & 0.2 & 0.5 & 0.3 \\
4 & 0.6 & 0.3 & 0.6 & 0.2 & 0.2 & 0.2 \\
\hline
\end{tabular}

